\title{
A Review of the Proposed Mechanistic Actions of Sodium Glucose Cotransporter-2 Inhibitors in the Treatment of Heart Failure
}

\author{
Brandon Nightingale
}

\begin{abstract}
Diabetes mellitus is a well-known risk factor for heart failure, and the reasons why are well understood. The incidence of diabetes mellitus is continuing to rise, posing a major concern in the medical world. The comorbidities associated with diabetes mellitus create a major hindrance on daily living, and promote the development of a plethora of other diseases. It is known that by controlling modifiable risk factors, such as glycemic control and body mass index, patients achieve more favorable outcomes. But, this is not always realistic and controlling modifiable risk factors should be balanced with a pharmacologic option. A relatively new drug class, which acts as an inhibitor of the sodium glucose cotransporter-2 receptor, has shown favorable outcomes in the treatment of heart failure associated with diabetes. However, the mechanism of action of this new drug class is not fully understood. There are several different proposed mechanisms of action for how sodium glucose cotransporter-2 inhibitors work in regards to the treatment of heart failure. This review will elaborate on those proposed mechanisms of action and offer insight into future implications of this relatively new drug class.
\end{abstract}

Keywords: Heart failure; Cardiovascular; Renal; Diabetes; SGLT2

\section{Historical Perspective}

Diabetes mellitus, both types 1 and 2, is largely associated with elevated blood glucose levels. In general, diabetes mellitus type 1 (DM1) is associated with a lack of insulin; whereas diabetes mellitus type 2 (DM2) is associated with insulin insensitivity. Regardless of the mechanistic approach behind each pathology, both DM1 and DM2 are associated with a plethora of comorbid conditions. Some of the noteworthy associations with diabetes are hypertension, hyperlipidemia and atherosclerosis, all of which are independent risk factors for

Manuscript submitted January 16, 2021, accepted January 27, 2021

Published online February 23, 2021

Saba University School of Medicine, Church Street, The Bottom, Saba, Dutch Caribbean. Email: b.nightingale@saba.edu

doi: https://doi.org/10.14740/cr1221 heart failure [1].

Historically, DM1 has been treated with insulin to combat the intrinsic lack of insulin in the body, and DM2 has been treated with oral glucose modulators to help increase the release of insulin and to increase insulin receptor sensitivity. The pharmacologic management of diabetes is dynamic with new medications regularly coming to market. The sodium glucose cotransporter-2 inhibitors (SGLT2i) are one of the newer drug classes created to combat DM2. The SGLT2i were first brought to market in 2013 for the treatment of DM2, and as of 2019 the Food and Drug Administration (FDA) has expanded the therapeutic scope to include heart failure with reduced ejection fraction. The following paragraphs will highlight some of the main features of this new drug class, as well as the specific SGLT receptors themselves.

The SGLT, which functions as symporters, are found throughout the body. Their main function is centered around the uptake of glucose, and they are dependent on an electrochemical gradient that is created by a downstream sodium potassium pump. There are six different types of SGLT receptors; however this paper will focus on the two most prevalent forms. The SGLT1 receptor is mainly found in the small intestine, and to a lesser extent in the later parts of the proximal renal tubule, and functions to facilitate the uptake of glucose. In contrast, the SGLT2 receptor is found only in the early parts of the proximal renal tubule where it functions to mediate the uptake of glucose. SGLT2 receptors are characterized by having a low affinity and a high capacity for glucose. This means that the SGLT2 receptors can handle a large amount of glucose before they become fully saturated. The reverse is true for SGLT1 receptors. SGLT2 receptors are also responsible for around $90 \%$ of glucose reabsorption, compared to about $10 \%$ for SGLT1 receptors [2].

The mechanism of action of SGLT2i is centered around the inhibition of SGLT2 receptors in the proximal convoluted tubules. This decreases the receptors ability to reabsorb glucose, thus promoting the excretion of glucose in the urine. This is beneficial to diabetic patients because as glucose is being eliminated through the urine, the plasma levels of glucose will decrease leading to an overall improvement in glycemic parameters. This effect is insulin independent, which means that hypoglycemia is an unlikely side effect of this drug class. A caveat to this drug class is that it relies on a person having relatively normal kidney function, meaning that if a person suffers from kidney failure then SGLT2i will have decreased 
efficacy [3].

As mentioned previously, heart failure is strongly associated with diabetes and is one of the leading causes of death in diabetic patients. Before the development of SGLT2i, there was not a diabetic drug that effectively combated this comorbidity without also causing severe side effects. There have been several studies to date that have clinically displayed the positive effects of SGLT2 $i$ in the treatment of heart failure. The overall mechanism of action is not fully understood, however there are several theories in place. Some of these theories are that SGLT2i improve volume status, such as preload and afterload, they help to improve cardiac metabolism, they have a direct effect on the sodium hydrogen exchange in the myocardium, and they have an inhibitory effect on cardiac fibrosis and adipokines. This paper will delve deeper into these proposed mechanistic actions and the overall impact that SGLT2i have on heart failure.

\section{Introduction}

Diabetes mellitus, a widespread and pervasive disease, has a significant impact on many different people throughout the world. The disease carries with it an increased risk of contracting many different comorbidities. Though our knowledge of the disease has become stronger throughout the years, the incidence and prevalence of diabetes mellitus has continued to rise. A study conducted in 2017 found that the prevalence of diabetes mellitus had risen by 102 million person years between 2005 and 2015, which is an increase of $30.6 \%$. The annual number of deaths due to diabetes mellitus also saw an uptick during this same timeframe, where deaths rose from 1.2 million to 1.5 million [4]. An article published in the Lancet, demonstrating the effects of several different diseases, showed that diabetes mellitus was one of the leading causes of years of life lost (YLL). In the year of 1990, diabetes mellitus was the 27th leading cause of YLL. It then rose to the 18th leading cause in 2005, before becoming the 15th leading cause in 2015. Ischemic heart disease continuously led the way as one of the leading causes of YLL, and held the title as the number one leading cause from 2005 to 2015 [5]. The statistics above present an alarming scenario, one being that the prevalence of diabetes mellitus is continuing to rise, and secondly the morbidity and mortality of the disease continues to be a major burden on society.

The complications of diabetes mellitus are vast, but include cardiovascular complications such as heart failure, diabetic nephropathy, gastroparesis, retinopathy and neuropathy. Diabetes mellitus confers one of the highest rates of coronary artery disease, which is one reason that blood pressure and low-density lipoprotein (LDL) levels are so tightly regulated [6]. Many of these complications can be associated with the increased rate of atherosclerosis associated with diabetes mellitus. The definitive reasons for the increased rate of atherosclerosis are not fully understood, but it is postulated that glycation of lipoproteins and increased platelet adhesiveness are two potential reasons [1]. A third reason, one which is more broad, is that diabetes mellitus creates a proinflammatory state, likely secondary to the release of adipokines from adipocytes, which aids in the progression of atherosclerosis, and can be attributed as a cause of plaque rupture [7].

Congestive heart failure (CHF), as mentioned above, is a major concern amongst diabetic patients. Several studies have been conducted to confirm this relationship. One of the more noteworthy studies, the Framingham Study, found that the incidence of diabetes in patients who suffered from CHF was $14 \%$ for men and $26 \%$ for women [8]. Another study was conducted between 1994 and 1999, and aimed to create a cohort of patients aged greater than 65 who had diabetes. Medicare claims were analyzed to assess the prevalence of heart failure in this population, and patients were followed to see if there were any new incidences of heart failure as well as to interpret the mortality of the disease. The study found that heart failure was prevalent in $22.3 \%$ of patients in 1994 and the incidence on developing heart failure between the years of 1995 - 1999 was 12.6 per 100 person-years. Mortality was highest in those who had heart failure, 32.7 per 100 person-years, versus those who did not suffer from heart failure, 3.7 per 100 person-years. An interesting caveat to these statistics is that the incidence of heart failure was highest in those patients who suffered from diabetes-related comorbidities. These comorbidities consisted of metabolic complications, ischemic heart disease, nephropathy, and peripheral vascular disease [9]. These numbers are alarming; based on this study, almost one out of every five diabetic patients with an age greater than 65 is likely to have CHF.

A retrospective cohort study that analyzed data from 8,231 patients with DM2 and 8,845 nondiabetic patients, who were similarly matched based on age and sex, aimed to give an updated outlook on how serious of a risk factor DM2 is for CHF. The study started following patients on January 1, 1997, and continued following them for 72 months. The study found that patients with DM2 had a much greater incidence of CHF than those without DM2 (incidence rate for diabetics was 30.9 cases per 1,000 person-years versus 12.4 cases per 1,000 person-years for nondiabetics). Modifiable risk factors were also shown to have a large implication in the development of CHF. Poor glycemic control and an elevated body mass index were two factors that were shown to be associated with a negative outcome. The rates of developing CHF were also greatest in younger patients, hinting that combating DM2 early in its course, is even more beneficial than previously thought [10]. Controlling the modifiable risk factors for DM2 is also extremely important as displayed by this study.

Controlling these modifiable risk factors will not fully eliminate the development of CHF in diabetic patients. Therefore, there needs to be a way to manage these patients from a pharmacological standpoint. Recently, in 2013, a new drug class, SGLT2i, was developed to treat DM2. This class works by inhibiting the SGLT2 receptor in the proximal renal tubule which decreases the receptors ability to reabsorb glucose. These drugs help to lower the total level of glucose in the body, thus helping with glycemic control. It was not until 2019 that the FDA approved SGLT2i for the treatment of CHF in diabetic patients. This was a major advancement in the drug world as there was not previously a well-established diabetic drug for the treatment of CHF. This review article will illicit the pro- 
posed mechanistic actions of this drug class for the treatment of CHF, and shed light onto future insight.

\section{Underlying Principles}

SGLT2i were originally developed for the treatment of DM2, with no implication in the treatment of heart failure. However, in 2019, the FDA approved dapagliflozin for the treatment of heart failure with reduced ejection fraction (HFrEF), creating a new niche for this drug class. Exactly how dapagliflozin and the other SGLT2i effectively combat heart failure is not entirely clear, but there have been several placebo based clinical trials displaying their efficacy in doing so.

With regards to treating heart failure, the exact mechanism of action is not known. However, there are several theorized mechanistic actions that may be responsible for these observed clinical outcomes, which will be discussed below. As with many pathologies, the intricate details are complex and are often linked with other comorbidities. It is important to keep in mind how one faulty measure can lead to another, creating a repeating cycle.

One proposed theory is that SGLT2i reduce sympathetic stimulation. The SGLT2 receptor has been hypothesized to engage in the activation of the sympathetic nervous system. When the sympathetic nervous system is over stimulated, the physiologic response is a chronic rise in blood pressure leading to hypertension, and eventual damage to the kidneys. One reason behind this is that sympathetic stimulation will cause the arterials to stiffen [11]. SGLT2i have been proved capable of improving endothelial function and aortic stiffness, and have also been shown to possibly induce vasodilation via activating voltage-gated-potassium channels and protein kinase $\mathrm{G}$ [12]. Both hypertension and kidney failure are associated with poorer outcomes in patients with heart failure. The kidney is a focal point for sympathetic overactivity, which is why targeting the SGLT2 receptor in the proximal renal tubule could offer therapeutic relief [11].

Another benefit of the SGLT2 $\mathrm{i}$ is their abilities to improve preload and afterload, ultimately improving ventricular functioning. An increased preload will allow the sarcomeres to stretch, thus increasing cardiac contractility. Afterload can be thought of as the amount of force the heart is working against. When afterload is reduced, the heart will have an easier time getting blood out to the rest of the body. The proposed mechanism for how SGLT2i accomplish this is that they alter natriuresis and glucosuria in the proximal renal tubule. This creates a state of osmotic diuresis. By promoting the excretion of sodium, the tubuloglomerular feedback system is stimulated, which causes afferent arteriolar vasoconstriction. This further reduces intraglomerular hypertension [12]. An interesting characteristic of SGLT2 $i$ is that they seem to selectively reduce interstitial fluid and not intravascular volume. By doing so it eliminates the body's neurohumoral reflexes which can be seen as an adverse effect of other diuretic agents [12].

As the heart begins to fail there is a diminished capacity for oxidizing fatty acids as fuel, one of the most prominent sources of energy for healthy cardiac tissue. Thus, the heart switches to oxidizing ketone bodies as an alternative source of energy. Beta-hydroxybutyrate dehydrogenase 1 (BDH1) is an essential enzyme in the oxidization of ketone bodies. This enzyme is upregulated in a heart undergoing hypertrophy as well as in a heart that is failing [13]. This elicits the importance of ketone bodies in heart failure, and paves way for the next hypothesized mechanism for how SGLT2i treat heart failure.

SGLT2 $\mathrm{i}$ help to improve cardiac efficiency and cardiac output by enhancing myocardial energetics and substrate efficiency [12]. As mentioned in the above paragraph, the failing heart will preferentially utilize ketone bodies for energy. SGLT2 $\mathrm{i}$ are able to exacerbate the production of ketone bodies, specifically beta-hydroxybutyrate (BOHB), offering the heart a surplus of energy rich nutrients. Increasing BOHB levels may also negate the effects of histone deacetylase which would prevent transcription pathways favoring hypertrophy from functioning properly. Also, the surge in ketone production would decrease the levels of acetyl-CoA in the body, which in turn would decrease hyperacetylation of mitochondria, which is harmful and hinders their energy producing abilities [12].

A proposed link between heart failure and diabetes mellitus is the sodium hydrogen exchanger (NHE) isotypes 1 and 3. NHE1 is found in myocardium and vasculature; and activation of this isotype will promote increased levels of cytosolic sodium and calcium. The rise in intracellular sodium, secondary to this specific isotype, has been associated with heart failure and cardiomyocyte injury. The NHE3 is found in the kidneys; and when this isotype is overexpressed it leads to increased sodium reabsorption. The increased sodium reabsorption will aid in the development of fluid retention and peripheral edema [14]. In summary, the deleterious effects of these isotypes are cardiac hypertrophy and damage, ultimately leading to heart failure, sodium retention which will lead to edema, and worsening of diabetic complications and glycemic control.

Both isoforms NHE1 and NHE3 are stimulated by insulin, glucose, and neurohumoral mechanisms. Insulin and glucose are both increased in patients with DM2, and neurohumoral mechanisms are increased in patients with heart failure [14]. Additionally, all three stimulants are entwined into a complex overall pathogenesis that sees each disease altering the other. For instance, if a patient has DM2 and heart failure and they suffer an exacerbation of their heart failure, they will ultimately see their diabetes suffer as well. Targeting these isotypes could offer therapeutic relief to both diabetic patients and those who are suffering from heart failure.

It is hypothesized that SGLT2i directly inhibit NHE1 in the myocardium, and promote natriuresis by causing the expression of NHE3 in the kidney to be decreased [12]. Thus, by being able to target both isotypes, SGLT2i could potentially be implicated in the management of both DM2 and heart failure independent of the other, meaning that if a patient has heart failure, and does not suffer from DM2, SGLT2i may still offer benefit.

Fibrosis, often regarded as irreversible damage, is a component common to heart failure. In severe forms of cardiomyocyte damage, the heart can begin to remodel, promoting a state of impaired ventricular functioning and ultimately accelerated heart failure. Being able to inhibit this process would be immensely significant in altering the progression of heart failure, 
and potentially aiding in the reversal of the disease. It is proposed that SGLT2i suppress collagen synthesis by activating M2 macrophages and inhibiting myofibroblast differentiation [12]. By doing so SGLT2i can enact antifibrotic effects, offering cardioprotective benefits. This theory is bolstered by findings from a lab study which demonstrated that SGLT2i increased STAT3 activity, STAT3 nuclear translocation, myocardial interleukin 10 (IL-10) levels, and the overall amount of M2 macrophage levels [15]. M2 macrophages are known to play a pivotal role in wound healing and tissue repair. The M2 macrophage accomplishes this by secreting IL-10, a powerful anti-inflammatory cytokine which helps to maintain normal tissue homeostasis.

Leptin, an adipokine that has a role in the regulation of sodium and cardiac inflammation and fibrosis, is known to be a key factor in the development of heart failure in patients with DM2. When leptin is synthesized, it enhances SGLT2 receptors in the kidney, causing further sodium retention. SGLT2i combat the effects of leptin in various ways. For instance, the SGLT2 receptors are blocked, promoting natriuresis. Secondly, the overall levels of leptin are diminished due to a decrease in the overall amount of per visceral adipose tissue [14]. Another benefit of SGLT2i use is that they increase the overall levels of adiponectin in the body. Adiponectin has been shown to reduce the overall amount of glucose produced in the liver. It also has an inhibitory effect towards tumor necrosis factor alpha (TNFalpha), an inflammatory cytokine. In terms of body weight, overall body fat, and basal plasma insulin levels, adiponectin has a negative correlation. This means that if adiponectin is increased, then overall body fat will be decreased [16].

\section{Current State of the Art}

There have been several clinical trials done that highlight the therapeutic effects of SLGT2i in the treatment of heart failure. The major clinical trials demonstrating this will be reviewed below. The intricate details of each clinical study will not be talked about; however the important clinical impacts discovered will be addressed. There are also a few ongoing clinical studies that will also be discussed.

The first cardiovascular outcome trial for SGLT2i was the Empagliflozin Cardiovascular Outcome Event Trial in Type 2 Diabetes Mellitus Patients-Removing Excess Glucose (EMPAREG OUTCOME trial). This was a randomized double-blind controlled trial that featured three groups. One group received a placebo daily, while the other two groups either received 10 $\mathrm{mg}$, or $25 \mathrm{mg}$, of empagliflozin daily. The primary composite outcome, which combines multiple outcomes into one measure, was death from cardiovascular causes, nonfatal myocardial infarctions, and nonfatal strokes. The primary composite outcome was analyzed between the empagliflozin groups combined, versus the placebo group. The secondary composite outcome was the primary outcome in addition to hospitalizations for unstable angina [17].

The EMPA-REG OUTCOME trial enrolled 7,020 patients with DM2 and established cardiovascular disease (CVD). The total number of patients enrolled into the two empagliflozin groups was 4,687, versus 2,333 patients in the placebo group. The primary composite outcome was observed in 490 patients $(10.5 \%)$ within the empagliflozin group, versus 282 patients $(12.1 \%)$ in the placebo group.

When analyzing the data, there was no major difference between the two groups for rates of myocardial infarction or stroke. There was also no difference between the two groups in regards to the secondary outcome. However, when compared to the placebo group, the empagliflozin group demonstrated significantly lower rates of deaths due to cardiovascular reasons $(3.7 \%$ vs. $5.9 \%, 38 \%$ relative risk reduction), hospitalization for heart failure $(2.7 \%$ vs. $4.1 \%, 35 \%$ relative risk reduction), and death from any cause $(5.7 \%$ vs. $8.3 \%, 32 \%$ relative risk reduction) [17]. Thus, this study clearly delineates the abilities of empagliflozin in the treatment of heart failure.

The second cardiovascular outcome trial featuring SGLT2i was the CANagliflozin CardioVascular Assessment Study (CANVAS), which combined two separate studies (the CANVAS and the CANVAS-Renal (CANVAS-R)) into one cohort. The setup of the study was a randomized double-blind controlled trial featuring two groups, a placebo group and a canagliflozin group. A total of 10,142 patients with diabetes, who also had high cardiovascular risks, were enrolled into the study and followed for a mean of 188.2 weeks [18].

The primary outcome of the study was a combined measure of deaths due to cardiovascular reasons, nonfatal myocardial infarctions, and nonfatal strokes. The canagliflozin group was found to have a lower rate of primary outcome when compared to the placebo group (26.9 vs. 31.5 participants per 1,000 patient-years) [19]. Hospitalizations secondary to heart failure, a secondary outcome measure, were also shown to be reduced in the canagliflozin group (5.5 vs. 8.7 participants per 1,000 patient-years). Although canagliflozin had favorable outcomes, as discussed above, it did not significantly reduce overall or cardiovascular mortality [20]. This unwanted outcome could be attributed to the diminished number of patients with established CVD at the beginning of the study ( $65.6 \%$ of study participants). Had this study included a higher baseline percentage of established CVD, as did the EMPA-REG OUTCOME trial, it may have had similar results regarding reducing overall or cardiovascular mortality.

The CANVAS study prompted a secondary analysis, Canagliflozin and Renal Events in Diabetes with Established Nephropathy and Clinical Evaluation (CREDENCE). This was a double-blind, randomized, clinical control trial consisting of two groups. One group received a placebo, while the other group received canagliflozin. A total of 4,401 patients were enrolled into the study, meeting the study criteria by having DM2 and albuminuric chronic kidney disease. The median follow-up period was roughly 2.62 years. Much like the CANVAS study, the overall number of patients with baseline ASCVD enrolled into CREDENCE was low (50.4\%) [20].

The primary composite outcome of the study was endstage kidney disease, a doubling of the serum creatinine lev$\mathrm{el}$, or death from kidney or heart related issues. Canagliflozin, when compared to the placebo, was superior in terms of lowering renal and cardiovascular risks, and the relative risk of the primary outcome was $30 \%$ lower in the canagliflozin group. When focusing on kidney specific issues, canagliflo- 
zin was again superior to the placebo; with the relative risk of end stage kidney disease, a doubling of the creatinine level, or death from kidney or heart related issues being lower by $34 \%$ in the canagliflozin group. There was also a lower risk of death due to cardiovascular issues, nonfatal myocardial infarctions, or stroke in the canagliflozin group. Hospitalizations secondary to heart failure were also diminished when using canagliflozin. Due to the overwhelming success of the study, CREDENCE was stopped early [21]. This study demonstrated that using canagliflozin positively influenced the outcomes in patients with DM2 and CKD.

The Dapagliflozin Effect on Cardiovascular EventsThrombolysis in Myocardial Infarction 58 trial (DECLARETIMI 58), aimed to evaluate the cardiovascular safety profile of dapagliflozin. The study was constructed as a randomized, double-blind clinical control trial, which featured a placebo group and a dapagliflozin group. A total of 17,160 patients were enrolled, with 6,974 patients having established atherosclerotic CVD (40.5\% of participants). Once assigned to a group, participants were followed for a median of 4.2 years [22].

The primary safety outcome for the study was a combination of major adverse cardiovascular events (MACE), which featured cardiovascular death, myocardial infarction, and ischemic stroke. Dapagliflozin did not effectively lower the rate of the primary safety outcome. As for the primary efficacy outcomes, MACE and the composite of cardiovascular death and hospitalization secondary to heart failure, dapagliflozin was not found to have a significant effect towards MACE, but did lower the rate of cardiovascular death and hospitalizations secondary to heart failure (4.9\% vs. 5.8\%; hazard ratio, $0.83)$. Secondary outcomes for the study were centered around a renal composite, consisting of estimated glomerular filtration rate (eGFR), new end-stage renal disease, and death from kidney or heart related issues [22].

The clinical trials discussed above, EMPA-REG OUTCOME, CANVAS, CREDENCE, and DECLARE-TIMI 58 have consistently demonstrated the potential implications for SGLT2 $\mathrm{i}$ in the treatment of heart failure in diabetic patients. There were minor discrepancies amongst the trials, but the resounding fact was that SGLT2i reduce the rate of hospitalizations secondary to heart failure and overall deaths related to cardiovascular issues. Although adverse events were not discussed in this review, SGLT2 $i$ are considered a safe therapeutic option.

\section{Key Limitations, Problems, Unaddressed Needs}

A potential problem may be the unknown implication of when to start patients on SGLT2i. The clinical trials mentioned above demonstrated that this drug class has therapeutic benefit for patients with, and without, atherosclerotic CVD, as well as selective benefit for kidney disease independent of heart failure. As more clinical studies are conducted, perhaps clearer guidelines will be put in place regarding starting of SGLT2i. Also, most of the clinical trials already conducted have param- eters set for kidney function, thus excluding many patients. It would be interesting to see how SGLT2 $i$ work across the spectrum, including patients with normal, diminished, and severely diminished kidney function.

Most studies were not carried out past 2 - 3 years, which may be due to ethical issues as the study groups often showed favorable outcomes. However, it would be beneficial to obtain long-term outcomes in patients on SGLT2i so that more safety measures and clinical guidelines can be put in place. Trials which compare the SGLT2i against other diabetic medications, displaying efficacy and adverse events would also be helpful in drawing comparison.

\section{Future Directions}

There are both ongoing studies, and studies that were recently completed, that are further evaluating the use of SGLT2i in the role of treating heart failure in diabetic patients. For example, Effect of Sotagliflozin on Cardiovascular and Renal Events in Patients With Type 2 Diabetes and Moderate Renal Impairment Who are at Cardiovascular Risk trial (SCORED), set out to evaluate the effects of sotagliflozin with respect to cardiovascular insult in diabetic patients. Sotagliflozin exerts its therapeutic action through the inhibition of both SGLT1 and SLGT2 receptors, making it the first dual SGLT inhibitor. Results have shown that sotagliflozin reduced the number of cardiovascular-related deaths, hospitalizations due to heart failure, and urgent visits related to cardiovascular causes [23]. Sotagliflozin was also shown to be effective in treating patients who recently suffered worsening heart failure. When administered before discharge, or soon thereafter, the drug was shown to decrease the risk of cardiovascular death and hospitalizations and urgent visits due to cardiovascular-related issues [24].

This drug offers a promising avenue towards possibly incorporating SGLT1 receptors in future therapeutic endeavors when targeting cardiovascular risks in diabetic patients. The positive outcomes associated with using a dual SGLT inhibitor are still not fully understood. One method may be that when SGLT2 receptors are inhibited, the burden put on the SGLT1 receptor for glucose transport is increased [25]. By targeting both receptors, SGLT1 and SGLT2, you can maximize the amount of control you have over the body's total glucose level. Another postulated therapeutic benefit of inhibiting the SGLT1 receptor is that it may reduce reactive oxygen species as the SGLT1 receptor is the only SGLT receptor expressed within cardiac tissue [26]. Future clinical trials should expand upon this success and increase the study populations to include patients who do not suffer from diabetes, but are impacted by CVD. There should also be an emphasis put on further evaluating the effect of SGLT2i in the treatment of heart failure independent of diabetes and chronic kidney disease.

\section{Conclusions}

Exactly how SGLT2i exert their cardioprotective benefits is 


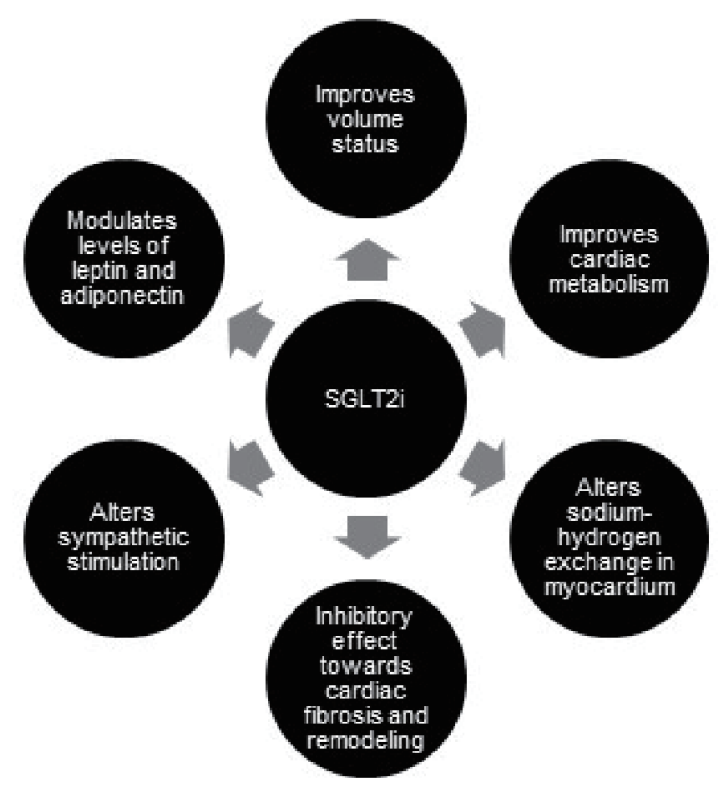

Figure 1. Proposed mechanistic actions of sodium glucose cotransorter-2 inhibitors (SGLT2i) in regards to treating heart failure.

still unclear. However, there are several postulated mechanisms of action which are summarized in Figure 1. The SGLT2i are thought to lower the risk factors for coronary events by improving hemodynamics, controlling sympathetic stimulation, inhibiting fibrosis and cardiac remodeling, improving cardiac efficiency and output, modulating the overall cytosolic sodium and calcium concentrations, and altering the adipokine levels.

Several clinical trials displayed these cardioprotective effects, demonstrating the efficacy of SGLT2i in treating heart failure in patients with DM2. Most notably, these drugs were shown to reduce the overall number of hospitalizations secondary to heart failure, and the overall number of cardiovascular deaths. The SGLT2i were also associated with a nephroprotective element, as they were shown to reduce the overall number of deaths related to renal complications.

The concluded clinical trials focused primarily on heart failure in patients with diabetes, and did not clearly separate the two diseases. Future studies should isolate the two conditions to gain an appreciated understanding of how SGLT2i work with respect to each disease independent of the other.

\section{Acknowledgments}

I would like to acknowledge Dr. James Bruzik for his continued support and guidance. Dr. Bruzik has been instrumental in helping me achieve my goals.

\section{Financial Disclosure}

None to declare.

\section{Conflict of Interest}

None to declare.

\section{Data Availability}

The author declares that data supporting the findings of this study are available within the article.

\section{References}

1. Agabegi ED, Duncan MD, Chuang K, Agabegi SS. Stepup medicine. Philadelphia: Wolters Kluwer. 2020.

2. Poulsen SB, Fenton RA, Rieg T. Sodium-glucose cotransport. Curr Opin Nephrol Hypertens. 2015;24(5):463-469.

3. Kalra S. Sodium Glucose Co-Transporter-2 (SGLT2) inhibitors: a review of their basic and clinical pharmacology. Diabetes Ther. 2014;5(2):355-366.

4. Ingelfinger JR, Jarcho JA. Increase in the incidence of diabetes and its implications. N Engl J Med. 2017;376(15):1473-1474.

5. G. B. D. Mortality. Causes of Death, Collaborators. Global, regional, and national life expectancy, all-cause mortality, and cause-specific mortality for 249 causes of death, 19802015: a systematic analysis for the Global Burden of Disease Study 2015. Lancet. 2016;388(10053):1459-1544.

6. Fischer C. Master the boards: Usmle Step 2 Ck. New York: Kaplan Publishing. 2019.

7. Freitas Lima LC, Braga VA, do Socorro de Franca Silva M, Cruz JC, Sousa Santos SH, de Oliveira Monteiro MM, Balarini CM. Adipokines, diabetes and atherosclerosis: an inflammatory association. Front Physiol. 2015;6:304.

8. Kannel WB, Hjortland M, Castelli WP. Role of diabetes in congestive heart failure: the Framingham study. Am J Cardiol. 1974;34(1):29-34.

9. Bertoni AG, Hundley WG, Massing MW, Bonds DE, Burke GL, Goff DC, Jr. Heart failure prevalence, incidence, and mortality in the elderly with diabetes. Diabetes Care. 2004;27(3):699-703.

10. Nichols GA, Gullion CM, Koro CE, Ephross SA, Brown JB. The incidence of congestive heart failure in type 2 diabetes: an update. Diabetes Care. 2004;27(8):1879-1884.

11. Sano M. A new class of drugs for heart failure: SGLT2 inhibitors reduce sympathetic overactivity. J Cardiol. 2018;71(5):471-476.

12. Verma S, McMurray JJV. SGLT2 inhibitors and mechanisms of cardiovascular benefit: a state-of-the-art review. Diabetologia. 2018;61(10):2108-2117.

13. Aubert G, Martin OJ, Horton JL, Lai L, Vega RB, Leone TC, Koves T, et al. The Failing Heart Relies on Ketone Bodies as a Fuel. Circulation. 2016;133(8):698-705.

14. Packer M. Activation and inhibition of sodium-hydrogen exchanger is a mechanism that links the pathophysiology and treatment of diabetes mellitus with that of heart failure. Circulation. 2017;136(16):1548-1559.

15. Lee TM, Chang NC, Lin SZ. Dapagliflozin, a selective 
SGLT2 Inhibitor, attenuated cardiac fibrosis by regulating the macrophage polarization via STAT3 signaling in infarcted rat hearts. Free Radic Biol Med. 2017;104:298310.

16. Wu P, Wen W, Li J, Xu J, Zhao M, Chen H, Sun J. Systematic Review and Meta-Analysis of Randomized controlled trials on the effect of SGLT2 inhibitor on blood leptin and adiponectin level in patients with type 2 diabetes. Horm Metab Res. 2019;51(8):487-494.

17. Zinman B, Wanner C, Lachin JM, Fitchett D, Bluhmki E, Hantel S, Mattheus M, et al. Empagliflozin, cardiovascular outcomes, and mortality in type 2 diabetes. N Engl J Med. 2015;373(22):2117-2128.

18. Kluger AY, Tecson KM, Lee AY, Lerma EV, Rangaswami J, Lepor NE, Cobble ME, et al. Class effects of SGLT2 inhibitors on cardiorenal outcomes. Cardiovasc Diabetol. 2019;18(1):99.

19. Neal B, Perkovic V, Mahaffey KW, de Zeeuw D, Fulcher G, Erondu N, Shaw W, et al. Canagliflozin and cardiovascular and renal events in type 2 diabetes. N Engl J Med. 2017;377(7):644-657.

20. Wojcik C, Warden BA. Mechanisms and evidence for heart failure benefits from SGLT2 inhibitors. Curr Cardiol Rep. 2019;21(10):130.
21. Perkovic V, Jardine MJ, Neal B, Bompoint S, Heerspink HJL, Charytan DM, Edwards R, et al. Canagliflozin and renal outcomes in type 2 diabetes and nephropathy. N Engl J Med. 2019;380(24):2295-2306.

22. Wiviott SD, Raz I, Bonaca MP, Mosenzon O, Kato ET, Cahn A, Silverman MG, et al. Dapagliflozin and cardiovascular outcomes in type 2 diabetes. N Engl J Med. 2019;380(4):347-357.

23. Bhatt DL, Szarek M, Pitt B, Cannon CP, Leiter LA, McGuire DK, Lewis JB, et al. Sotagliflozin in patients with diabetes and chronic kidney disease. N Engl J Med. 2021;384(2):129-139.

24. Bhatt DL, Szarek M, Steg PG, Cannon CP, Leiter LA, McGuire DK, Lewis JB, et al. Sotagliflozin in patients with diabetes and recent worsening heart failure. N Engl J Med. 2021;384(2):117-128.

25. Cefalo CMA, Cinti F, Moffa S, Impronta F, Sorice GP, Mezza T, Pontecorvi A, et al. Sotagliflozin, the first dual SGLT inhibitor: current outlook and perspectives. Cardiovasc Diabetol. 2019;18(1):20.

26. Kalra J, Mangali SB, Dasari D, Bhat A, Goyal S, Dhar I, Sriram D, et al. SGLT1 inhibition boon or bane for diabetes-associated cardiomyopathy. Fundam Clin Pharmacol. 2020;34(2):173-188. 